http://jmscr.igmpublication.org/home/ ISSN (e)-2347-176x ISSN (p) 2455-0450 crossref DOI: https://dx.doi.org/10.18535/jmscr/v7i12.21

Original Research Article

\title{
Study of Evaluation of Platelet Count among the patient suffering from Dengue Fever Attending in Tertiary Care Hospital at N.M.C.H., Patna
}

\author{
Authors \\ Dr Arun Kumar ${ }^{1}$, Dr Manohar Lal ${ }^{2 *}$, Dr O. P. Dwivedy \\ ${ }^{1}$ Tutor, Department of Pathology, Nalanda Medical College, Patna \\ ${ }^{2}$ Assistant Professor, Department of Pathology, Nalanda Medical College, Patna \\ ${ }^{3}$ Professor and HOD, Department of Pathology, Nalanda Medical College, Patna \\ *Corresponding Author \\ Dr Manohar Lal \\ Assistant Professor, Department of Pathology, Nalanda Medical College, Patna, India
}

\begin{abstract}
Objective: Dengue is one of the most prevalent mosquito's borne arboviral infections in India. Seventy percent of the 96 million apparent infections occur in Asia, in which India is making up to one third of the total. Dengue fever has emerged, spread and established itself rapidly despite considerable efforts to control the mosquito populations, Aim of present study was to evaluate the platelet count of patients suffering from Dengue Fever.

Material and Methods: A total of 46 patients with different sex and age groups were attending in the medical OPD and IPD with clinical features of dengue infection and were dengue serologically positive were included in the study. After thorough clinical history, all the patients were subjected to proper clinical examination and referred to our department for platelet count $(C B C)$. Platelet count was done by fully automated five part CBC machine supplied by Mindray. Platelet count was also manually evaluated.

Results: Out of the 46 dengue serological positive patients, 20 (43.48\%) patients were belonged in the age group of 15 - 30 years, followed by less than 15 years of age group which was 9 (28.26\%), 31 cases (67.39 $\%)$ were males and $15(32.61 \%)$ cases were females. Out of 46 patients, 28 (60.80\%) patients had mild thrombocytopenia (platelet count 51,000 - 1 lakh), 7 cases $(15.21 \%)$ had moderate thrombocytopenia (platelet count 20,000 -50,000), 4 cases (8.69\%) had severe thrombocytopenia (platelet count less than 20,000), and the remaining 7 cases (15.21\%) had platelet count more than 1 Lakhs. A statistically significant association was found between the age groups and severity of thrombocytopenia.

Conclusion: This retrospective study highlighted the drastic fall in the platelet count which life is threatening. More studies in this regard could further reveal the correlation between platelet count and dengue outbreaks, which would help in making the strategies and plans to forecast any outbreak in future well in advance.

Keywords: Dengue infection, Platelet count, Dengue hemorrhagic fever, Thrombocytopenia.
\end{abstract}

\section{Introduction}

Dengue is a mosquito borne arboviral infection prevalent worldwide. About 100 million cases of
Dengue Fever (DF) and half million cases of Dengue Hemorrhagic Fever (DHF) occurs worldwide annually. Epidemics of DF and DHF in 
India were seen regularly since 1989 but now dengue is endemic in India. From previous few years there was a major outbreak of dengue in Patna, Bihar. During the previous few years, the spectrum of dengue fever in India appears to be changed. Children were predominantly affected a decade ago, but in recent years doctors have seen an increasing numbers of adult dengue patients, with increasing morbidity and numbers of adult deaths due to dengue. Dengue fever is an acute febrile illness caused by four serotypes of Dengue virus and characterized a spectrum of disease, biphasic fever, myalgia, arthralgia, rash and Leucopoenia. Dengue hemorrhagic Fever (DHF) is characterized by hemoconcentration, abnormality of hemostasis and in severe cases by a fluid and protein losing shock syndrome (Dengue Shock Syndrome or DSS). In majority of patients thrombocytopenia is transient and asymptomatic but in significant number of cases there is bleeding manifestations. Spontaneous bleeding is noted in platelet count of Less than 20,000 in majority of patients. Petechae or purpura is seen in platelet count in the range of 20,000-40,000. This signifies the need to evaluate platelet count and the follow up after platelet transfusion.

\section{Material and Methods}

The present study was conducted in the Department of Pathology, Nalanda Medical College, Patna with the help of Department of Microbiology, Medicine, and Pediatrics during the period of July 2018 to September 2019.
A total of 46 patients who were serologically positive for Dengue infection were admitted in our hospital. After thorough clinical history, all the patients were subjected to proper clinical examination and referred to our department for platelet count (CBC) and from all the patients Platelet count was done, repeated regularly every day during the hospital stay. Platelet count was done by fully automated five part CBC machine supplied by Mindray. Platelet count was also manually evaluated. Patients with thrombocytopenia but serologically negative were not included in the study.

\section{Result}

Out of the 46 dengue serology positive patients, $20(43.48 \%)$ patients were belonged in the age group of 15 - 30 years, followed by less than 15 years of age group which was $9(28.26 \%), 31$ cases $(67.39 \%)$ were males and $15(32.61 \%)$ cases were females. Out of 46 patients, 28 $(60.80 \%)$ patients had mild thrombocytopenia (platelet count 51,000 - 1 lakh), 7 cases (15.21\%) had moderate thrombocytopenia (platelet count $20,000-50,000), 4$ cases $(8.69 \%)$ had severe thrombocytopenia (platelet count less than 20,000), and the remaining 7 cases $(15.21 \%)$ had platelet count more than 1 Lakhs.

Thrombocytopenia was found to be more severe in age group of less than 15 years, when compared to other age groups. A statistically significant association was found between the age groups and severity of thrombocytopenia.

Table-1 shows Age and Sex distribution of Dengue fever patients

\begin{tabular}{|l|c|c|c|c|}
\hline \multirow{2}{*}{ Age Groups of patients in years } & \multicolumn{4}{|c|}{ Sex of patients (n=46) } \\
\cline { 2 - 5 } & Male & Female & Total & Percentage \\
\hline Less than 15 & 9 & 4 & 13 & 28.26 \\
\hline $\mathbf{1 5 - 3 0}$ & 14 & 6 & 20 & 43.48 \\
\hline $\mathbf{3 1 - 5 0}$ & 5 & 3 & 8 & 17.39 \\
\hline More than 50 & 3 & 2 & 5 & 10.87 \\
\hline Total no. patients & $31(67.39 \%)$ & $15(32.61 \%)$ & 46 & $100 \%$ \\
\hline
\end{tabular}


Table-2 shows distribution of Platelet Count according to age group

\begin{tabular}{|l|c|c|c|c|}
\hline \multirow{2}{*}{$\begin{array}{l}\text { Age Groups of } \\
\text { patients in years }\end{array}$} & \multicolumn{4}{|c|}{ Platelet Count in per microLiter } \\
\cline { 2 - 5 } & Less than 20000 & $21000-50000$ & $51000-100000$ & More than 100000 \\
\hline Less than 15 & 2 & 3 & 6 & 2 \\
\hline $\mathbf{1 5 - 3 0}$ & 1 & 2 & 14 & 3 \\
\hline $\mathbf{3 1 - 5 0}$ & 0 & 1 & 6 & 1 \\
\hline More than 50 & 1 & 1 & 2 & 1 \\
\hline Total & $4(8.69 \%)$ & $7(15.21 \%)$ & $28(60.86 \%)$ & $7(15.21 \%)$ \\
\hline
\end{tabular}

\section{Discussion}

In the present study, Dengue Serology positive patients were more affected in the age group of 15-30 years and this is in accordance with the study by Pruthvi et al. In Pakistan study by Erum et al. shows median age group affected by dengue infections are young patients and males were affected predominantly. In our study Dengue was predominant among males $(67.39 \%)$ than females $(32.61 \%)$. This is in accordance with the study by Jayashree et al. The dengue virus induced bone marrow suppression decreased platelet synthesis, an immune mechanism of thrombocytopenia caused by increased platelet destruction appears to be operative in patients with DHF.

\section{Conclusion}

This study highlighted a drastic fall in the platelet count which is life threatening and may lead to bleeding episodes if close monitoring of platelet count is not assured. Median age group of Dengue patients has decreased and younger patients have become more susceptible. The only way to prevent from infection and developing Dengue fever is to avoid being bitten by the mosquito. Further research needed about the significant dengue epidemic during climatic conditions.

\section{References}

1. Swash M (1995) Doctor and patient, Hutchison's Clinical Methods (20 $0^{\text {th }} \mathrm{edn}$ ).

2. Rajajee S (1995) Dengue Hemorrhagic fever, the need for awareness. IAP $J$ Practical Pediatric 2: 131-135.

3. Handian RI (2001) Bleeding and thrombosis. Harrison principles of internal medicine $\left(18^{\text {th }}\right.$ edn $)$, McGraw Hill, USA.
4. Pruthvi D, Shashikala P, Shenoy V. Evaluation of platelet count in dengue fever along with seasonal variation of dengue infection. J Blood Disorders Transf. 2012;3:128.

5. Khan E, Kisat M, Khan N, Nasir A, Ayub $S$, et al. Demographic and clinical features of dengue fever in Pakistan from 20032007: A retrospective cross-sectional study, plos one 2010;5(9):e125-05.

6. Jayashree K, Manasa GC, Pallavi P. Indian Journal Hematology Blood Transfusion. 2011;27(3):127-30

7. De Castro RA, de Castro JA, Barez MY, Frias MV, Dixit J, Genereux M. Thrombocytopenia associated with dengue hemorrhagic fever responds to intravenous administration of anti-D ( $\mathrm{Rh}$ (o) -D) immune globulin. Am J Trop Med Hyg.2007; 76(4):737-42.

8. Text book of medical microbiology by Ananthanarayan and panikar $10^{\text {th }}$ edition. 